\title{
Growth Anisotropy and Pattern Formation in Metal Epitaxy
}

\author{
Louis C. Jorritsma, ${ }^{1}$ Matthieu Bijnagte, ${ }^{1}$ Georg Rosenfeld ${ }^{2}$ and Bene Poelsema ${ }^{1}$ \\ ${ }^{1}$ Faculty of Applied Physics and Centre for Materials Research, University of Twente, P.O. Box 217, \\ NL-7500 AE Enschede, The Netherlands \\ ${ }^{2}$ Institut für Grenzflächenforschung und Vakuumphysik, Forschungszentrum Jülich, D-52425 Jülich, Germany
}

(Received 17 October 1996)

\begin{abstract}
Evidence for the formation of growth induced, ordered checkerboardlike arrangements of mesas has been obtained. These patterns develop on a metal substrate with square symmetry after deposition of tens of monolayers. Its origin is traced back to laterally anisotropic advance rates of island edges in combination with slope selection. The foundation for the mesa arrangement is already laid just after coalescence of the adatom islands in the first monolayer. The results are exemplified in a high resolution surface diffraction study for the growth of $\mathrm{Cu}$ on $\mathrm{Cu}(001)$. [S0031-9007(97)02309-0]
\end{abstract}

PACS numbers: 68.55.Jk, 61.14.Hg, 68.35.Bs, 81.10.-h

The formation of ordered structures on surfaces during epitaxial growth on surfaces has recently obtained much attention. One prominent result of studies in this field is that in a nonequilibrium system diffusion controlled growth may lead to well defined dendritic patterns $[1,2]$. Particularly nice examples of this phenomenon have recently been reported by both Hohage et al. [3] and Brune et al. [4] for metal islands on metal fcc (111) substrates. Like fractals, these islands have a branched, rugged structure. In contrast to fractals, the branches develop along preferred directions, i.e., along $\langle 110\rangle$ directions on the (111)-oriented substrates, leading eventually to triangular envelopes of the ramified islands. The formation of dendrites rather than fractals, i.e., of orientationally ordered instead of random island structures, has been traced back to strong diffusion anisotropies at the extremities of the branches. This applies, in particular, to an asymmetric diffusion rate of atoms around island corners [3,4]. In this Letter we present evidence for the fact that diffusion anisotropy not only leads to ordering in the twodimensional regime, but that this interrelationship applies more generally. We show for the growth of tens of monolayers (ML) of $\mathrm{Cu}$ on $\mathrm{Cu}(001)$ that also ordering in three dimensions occurs due to growth anisotropies. In particular, our high-resolution low energy electron diffraction data reveal the formation of a checkerboard pattern existing of $\mathrm{Cu}$ mesas with quite well defined slopes. This self-organization occurs in a broad temperature regime in which both the average distance between the mesas and their preferred slopes depend distinctly on the substrate temperature. As a remarkable and important phenomenon we emphasize that the base structure develops already immediately after coalescence in the first monolayer at a deposit of approximately $0.7 \mathrm{ML}$ (monolayer). Our results are believed to apply in general for square substrate orientations.

The experiments have been performed in a home-built $\mathrm{He}$ atom diffraction (TEAS) apparatus equipped with an Omicron high-resolution SPALEED (spot profile analysis low energy electron diffraction) instrument, an ion gun, a CMA-Auger system, and several deposition facilities. The transfer widths of the SPALEED-and the TEAS device amount to about 1500 and $500 \AA$, respectively. The ex situ desulfurized $\mathrm{Cu}$ crystal has been further prepared in UHV by numerous cycles of sputtering with $800 \mathrm{eV} \mathrm{Ar}^{+}$ions and prolonged heating at about $1000 \mathrm{~K}$. This procedure allows standard mean terrace widths well exceeding $1000 \AA$. Copper has been sublimated from a thoroughly desulfurized disk, heated from the rear by means of electron bombardment. The growth experiments described in this Letter have been performed mostly at a substrate temperature of $\sim 250 \mathrm{~K}$ and with a deposition rate of about $0.01 \mathrm{ML} / \mathrm{s}$. Immediately after deposition the temperature of the sample has been quenched rapidly in order to suppress undesired diffusion. The SPALEED data have been acquired with the substrate held at $100 \mathrm{~K}$. The profiles of the specular peak (higher order peaks behave similarly) have been taken near destructive interference conditions.

Figure 1 displays a representative example of the profile of the specular SPALEED spot acquired after deposition of tens of monolayers of copper. In this case the deposit amounts to about $20 \mathrm{ML}$. The profile shows an extremely rich structure. The intensity at the Bragg position exhibits a pronounced minimum. This indicates that many layers are exposed to the probing electron beam: the growth proceeds in a multilayer fashion revealing restricted interlayer diffusion (see below, Fig. 3). The spot profile exhibits four well developed maxima (lobes), peaked roughly in the $\langle 100\rangle$ azimuthal directions. The general aspect of the image is reminiscent of some earlier published data $[5,6]$, which were obtained for the same growth system. The obtained spot profile thus shows a well developed fourfold symmetry as should be expected for the $\mathrm{Cu}(001)$ surface, which is illustrated best by the contour plot in the inset. For low intensities the contour assumes a fourfold symmetric shape. As is well known from textbooks on diffraction, they are indicative of a 


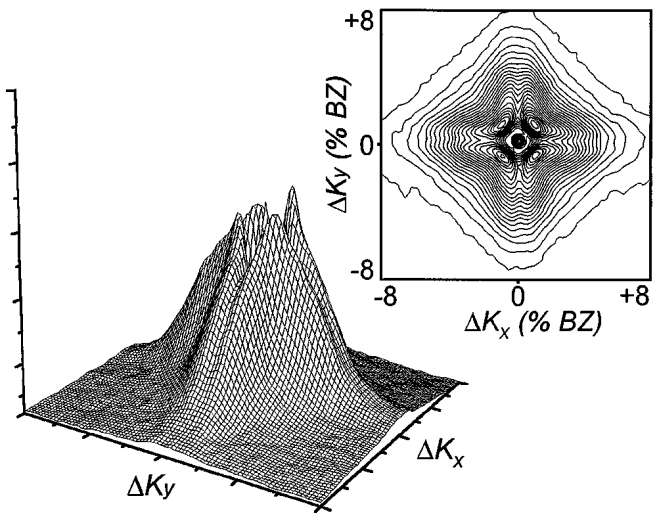

FIG. 1. Profile of the specular LEED spot after deposition of $20 \mathrm{ML} \mathrm{Cu}$ at a rate of $\sim 0.01 \mathrm{ML} / \mathrm{s}$ with the substrate at $246 \mathrm{~K}$. Inset: contour plot of the same data.

square shape of the adatom islands (see Fig. 4 and discussion). The ledges of the adatom island structures are preferentially oriented along the close packed $\langle 110\rangle$ directions. The pronounced four-lobed structure demonstrates the presence of strong order along the $\langle 100\rangle$ directions, i.e., along the diagonals of the surface projected adatom islands. A particularly interesting feature is the appearance of a previously unnoticed feature in the diffraction spot profile: the evolution of couples of well developed parallel ridges along the $\langle 110\rangle$ directions. These ridges are quite clear in the contour plot. They appear as maxima at distances which do not depend on the parallel components of the wave vector change, $\Delta k_{x}$ and $\Delta k_{y}$. These well developed ridges occur in a broad temperature range, roughly varying from 100 to $300 \mathrm{~K}$ and in a wide coverage regime $(\sim 15-40 \mathrm{ML})$. Their separation becomes increasingly smaller with increasing substrate temperature. These well developed ridges represent the key evidence for the formation of a checkerboardlike arrangement of copper mounds. The separation between the ridges relates reciprocally to a distance scale between the centers of the mounds. We think of a mound as being a mesa, i.e., a flattened pyramid the slope of which becomes gradually better defined with increasing deposition. In order to better understand these features we made a few qualitative calculations.

Figure 2(b) shows a cartoon of idealized pyramids arranged on a perfect checkerboard. Their base plates have a square shape. Figure 2(a) shows a contour plot of the calculated coherent diffraction pattern, under destructive interference conditions, from this arrangement of pyramids. One obtains four couples of closely separated spots; their positions are determined by the ideal superlattice on which the pyramids are based. Thus the distance between the spots is reciprocally related to the distance between the pyramid centers. The number of diffraction spots with appreciable intensity will be determined by the shape of the terraces. For ideal pyramids this implies that per facet (a)

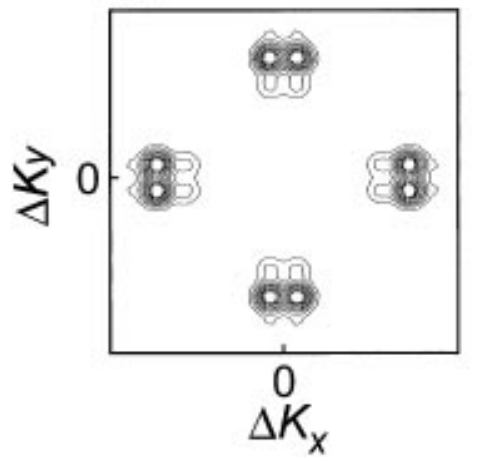

(b)

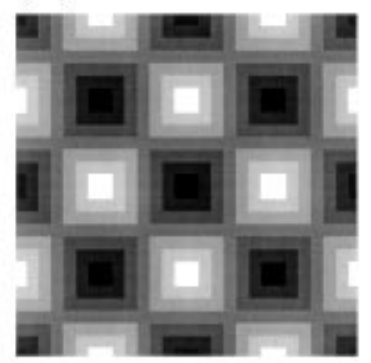

FIG. 2. Calculated specular spot profile (a) for diffraction from a checkerboardlike arrangement of regular pyramids (b). Grey scale refers to various terraces.

orientation, roughly speaking, only two spots will carry measurable intensity. In order to mimic the reality on the surface more closely one has to adopt distributions with finite widths both for the slope and the lateral separation. For a finite distribution of slopes of the facets one should therefore expect the peaks to lie on two parallel lines. So from a comparison of the calculated result and the experimental peak profile one immediately concludes that the slope of the developing pyramids has a rather broad distribution as one should expect for developing mesas. In addition, the checkerboard pattern will not be as ideal as in the cartoon. The introduction of some lateral disorder leads to a broadening of the diffraction spots. The overall result of introducing statistics leads eventually to the parallel ridges appearing in the experimental diffraction spot profile.

Upon further deposition (around 80-100 ML) we also observe facet peaks in the diffraction pattern (not shown in the framework of this Letter). In close agreement with Refs. [7] and [8] we find (113), (115), and (117) facets depending on the substrate temperature: below about $180 \mathrm{~K}$, between about 180 and $280 \mathrm{~K}$, and between about 280 and $300 \mathrm{~K}$, respectively. At and beyond this point the stripes in the spot profiles start to disappear, beginning at large $\Delta k_{\|}$values (i.e., at small length scales).

Our data appear to be also consistent with earlier theoretical results obtained by Plischke and Siegert $[9,10]$. These authors have, triggered by the growth instabilities for $\mathrm{Cu} / \mathrm{Cu}(001)$ observed by Ernst et al. [7,8], proposed a recipe, which leads to slope selection, i.e., to the growth of pyramidlike structures, under typical conditions of molecular beam epitaxy. Important ingredients in this description are two postulations artificially introducing both lateral growth anisotropy and slope selection. The first gives rise to the growth of square islands, while the latter takes account for the evolution of slopes. Simulations led to the formation of pyramids arranged on a 
checkerboardlike pattern $[9,10]$. Our present experimental results are to a high degree consistent with their results. A consequence of this scenario is an increase of the separation between the pyramids at large depositions. Indeed, Stroscio et al. [11] have shown for the system $\mathrm{Fe} / \mathrm{Fe}(001)$ that at large depositions the characteristic separation between the moundlike structures increases. This is a well accepted feature of a growth instability: Finally only one huge pyramid would be observed. Thus ultimately after full development of the facets the smaller mounds will be overgrown and the checkerboard pattern will gradually disappear. Such behavior is consistent with our observations as mentioned above. Below we address the issue of which mechanism is the key contributor to the formation of the checkerboard pattern. It turns out that the basic ingredients are already present just after coalescence of the submonolayer adatom islands. For this purpose we will closely inspect the initial growth behavior.

Figure 3 shows the height of the specular He peak, measured under destructive interference conditions, during the deposition of copper. Initially the He-peak height oscillates revealing quasi layer-by-layer growth $[12,13]$. This result agrees with data obtained previously by various authors [14-18]. The oscillations are strongly damped, indicative of restricted interlayer mass transport and finally even disappear revealing a crossover to a quasimultilayer growth regime. The latter growth mode implies that nucleation in the next layer takes place before coalescence occurs $[12,19,20]$. This behavior requires that interlayer diffusion is hampered by the existence of an Ehrlich-Schwoebel barrier hindering the descent of adatoms onto a lower terrace. We will concentrate next on the development of the surface morphology as a result of submonolayer deposition where the growth essentially proceeds in a two dimensional fashion (cf. Fig. 3).

Figure 4(a) shows a contour plot of the specular peak obtained after deposition of 0.5 monolayer copper. The measured peak profile constitutes a nice example of an electron diffraction analog of optical Fraunhofer diffraction from square apertures [21]. The structureless sharp ring around the specular peak indi-

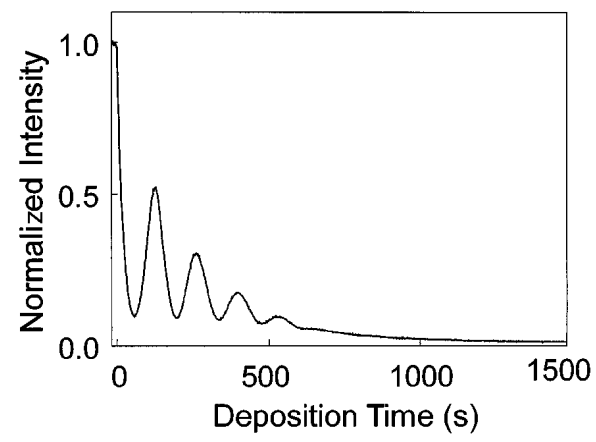

FIG. 3. He-specular peak height during deposition of $\mathrm{Cu}$ with the substrate at $250 \mathrm{~K}$. cates that the separation between adatom islands has a narrow distribution and reveals the absence of azimuthal preference for finding neighboring islands. The fourfold structure outside the ring reflects the size distribution and the dominant orientation of the square islands. Global inspection of the contour plot immediately leads to the conclusion that the steps of the square adatom islands are preferentially oriented along $\langle 110\rangle$ directions. The formation of these preferentially square shaped islands, even at relatively low substrate temperatures, indicates that the barrier for diffusion of adatoms along the $\langle 110\rangle$-oriented ledges is low. Note that the island size distribution does not affect the ring shape in this peak profile. The existence of square adatom and vacancy islands on $\mathrm{Cu}(001)$ has recently been observed also with STM $[22,23]$.

We consider now the peak profile obtained after deposition of 0.7 monolayers copper shown in Fig. 4(b). This image is representative for the situation just after coalescence of the adatom islands in the first layer. The peak profile now shows a pronounced fourfold symmetry, not only in the fourfold symmetric structure outside the ring, but also in the ring itself: The ring has maxima in the $\langle 100\rangle$ directions. While the step edges are still oriented in the $\langle 110\rangle$ direction, the initially isotropic distribution of island separations has changed into a distribution which is peaked in the $\langle 100\rangle$ directions. Thus the preference for ordering along $\langle 100\rangle$, which prevails at much higher coverages (Fig. 1) and is an important factor in the development of the checkerboard pattern, is already present directly after coalescence of the islands in the first monolayer. The reason for this phenomenon is easy to explain. Obviously, the reordering which could take place as a result of coalescence during deposition conditions is difficult to describe. Very likely the anisotropic island edge advance rates play a decisive role: The equilibrium shaped square islands grow in the $\langle 100\rangle$ direction $\sqrt{2}$ times faster than in the $\langle 110\rangle$ direction. Since the initial separation between islands is isotropic, coalescence of the adatom islands takes place preferentially along their diagonals, i.e., along $\langle 100\rangle$ azimuths. The details of the subsequent course of events have to be analyzed further. A possible scenario might be that the remaining vacancy (a) $0.5 \mathrm{ML}$

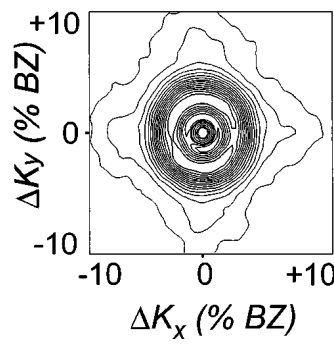

(b) $0.7 \mathrm{ML}$

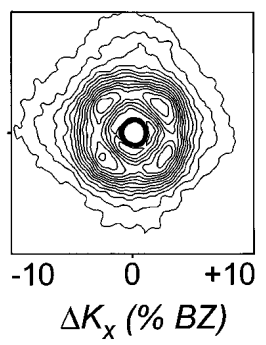

FIG. 4. Contour plots of specular LEED spot after deposition of 0.5 (a) and $0.7 \mathrm{ML}$ (b) $250 \mathrm{~K}$. 
clusters (i.e., yet uncovered areas of the original substrate) will tend to assume a square equilibrium shape, reducing the order along $\langle 110\rangle$ directions. The preference for correlations of adatom structures along the diagonal $(\langle 100\rangle)$ directions is even reinforced when nucleation in the second layer takes place. This has to take place in the coverage interval between the onset of coalescence and deposition of a monolayer equivalent. The precise moment depends on the height of the Ehrlich/Schwoebel barrier, the substrate temperature, and the deposition rate.

As a consequence of the above outlined scenario for the evolution of the growth front the separation of the ridges (see Fig. 1) must be intimately related to the length scale already set during the initial nucleation stage on the freshly prepared surface. Figure 5 shows an independent check of this feature: It shows a plot of the log of the ridge separation and of the $\Delta k_{\|}$values corresponding to the maximum of the ring structure [Fig. 4(a)] versus the reciprocal substrate temperature. Indeed the slopes are identical and, moreover, agree nicely with those found previously $[5,6,7,16]$. A closer inspection shows that the quantitative length scales also agree nicely.

In summary, we have shown that isotropic surface diffusion of single adatoms, in combination with the forma-

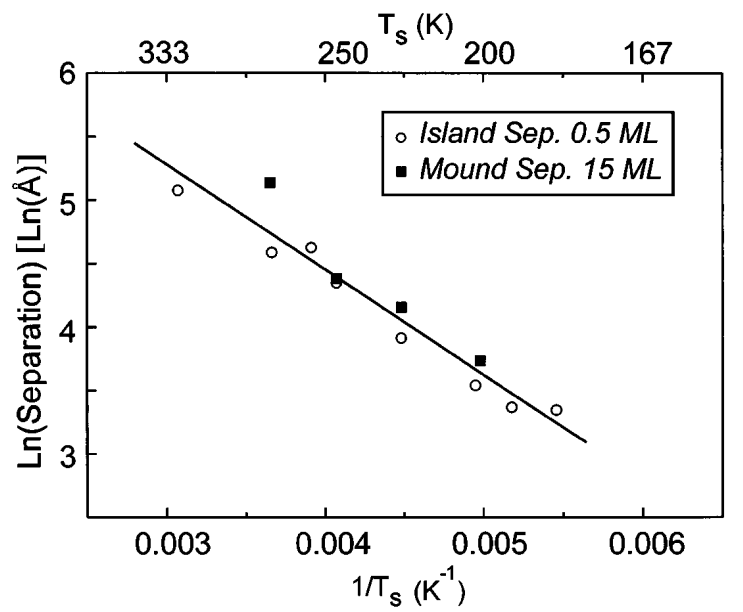

FIG. 5. FWHM of the ring structure $(O)$ and the separation of the stripes ( $\boldsymbol{\square})$ (see text) after deposition of 0.5 and $15 \mathrm{ML}$, respectively, versus the reciprocal substrate temperature. tion of equilibrium shaped (square) adatom islands leads to laterally anisotropic growth rates. Combined with a finite Ehrlich-Schwoebel barrier this gives rise to the formation of checkerboard patterns of $\mathrm{Cu}$ mesas on $\mathrm{Cu}(001)$. The base for this ordering phenomenon in tens of monolayers thick deposits is already laid just after coalescence of the growing adatom islands in the first monolayer. The observed features are believed to be a general property of unreconstructed (001) surfaces whenever the three conditions mentioned above are fulfilled.

[1] Y. Sawada, A. Dougherty, and J. P. Gollub, Phys. Rev. Lett. 56, 1260 (1986).

[2] D. Grier et al., Phys. Rev. Lett. 56, 1264 (1986).

[3] M. Hohage et al., Phys. Rev. Lett. 76, 2366 (1996).

[4] H. Brune et al., Surf. Sci. 349, L115 (1996).

[5] H. Dürr, J.F. Wendelken, and J.-K. Zuo, Surf. Sci. 328, L527 (1995).

[6] G. L. Nyberg, M. T. Kief, and W.F. Eglehof, Jr., Phys. Rev. B 48, 14509 (1993).

[7] H.-J. Ernst et al., J. Vac. Sci. Technol. A 12, 1809 (1994).

[8] H.-J. Ernst et al., Phys. Rev. Lett. 72, 112 (1994).

[9] M. Siegert and M. Plischke, Phys. Rev. Lett. 73, 1517 (1994).

[10] M. Plischke and M. Siegert, Phys. Rev. E 53, 307 (1996).

[11] J. A. Stroscio et al., Phys. Rev. Lett. 75, 4246 (1995).

[12] B. Poelsema et al., Appl. Phys. A 53, 369 (1991).

[13] B. Poelsema and G. Comsa, Springer Tracts in Modern Physics (Springer, Heidelberg, 1989), Vol. 115.

[14] L. J. Gomez et al., Phys. Rev. B 31, 255 (1985).

[15] W. F. Engelhoff, Jr. and I. Jacob, Phys. Rev. Lett. 62, 921 (1989).

[16] H.-J. Ernst, F. Fabre, and J. Lapujoulade, Surf. Sci. 275, L682 (1992).

[17] H.-J. Ernst, F. Fabre, and J. Lapujoulade, Phys. Rev. B 46, 1929 (1992).

[18] H. A. van der Vegt et al., Phys. Rev. B 51, 14806 (1995).

[19] G. Rosenfeld et al., Appl. Phys. A 61, 455 (1995).

[20] J. Tersoff, A. W. Denier van der Gon, and R. M. Tromp, Phys. Rev. Lett. 72, 266 (1994).

[21] E. Hecht and A. Zajac, Optics (Addison-Wesley, Reading, 1974), Chap. 10.

[22] J. C. Girard et al., Surf. Sci. 302, 73 (1994).

[23] M. Ritter et al., Surf. Sci. 348, 243 (1996). 